\title{
Light-front quantum mechanics and quantum field theory
}

\author{
W. N. Polyzou*t \\ The University of Iowa \\ E-mail: polyzoucuiowa.edu
}

This contribution discusses some of the advantages and unique properties of relativistic quantum theories with kinematic light-front symmetries.

Light Cone 2019 - QCD on the light cone: from hadrons to heavy ions - LC2019

16-20 September 2019

L'École Polytechnique, Palaiseau, France

* Speaker.

${ }^{\dagger}$ This research supported by the US Department of Energy, Office of Science, grant number DE-SC0016457 


\section{Introduction}

In 1939 E.P. Wigner [1] showed that a necessary and sufficient condition for quantum observables, (probabilities, expectation values, and ensemble averages) to have the same values in all inertial coordinate systems is the existence of a unitary ray representation of the component of the Poincare group connected to the identity. In order to satisfy the commutation relations involving boost and translational generators,

$$
\left[K^{i}, P^{j}\right]=i \delta_{i j} H
$$

interactions must appear in at least three of the Poincaré generators.

In 1949 P.A.M. Dirac [2] identified three representations of a relativistic dynamics with the largest interaction independent subgroups of the Poincaré group. The kinematic subgroups identified by Dirac include the Lorentz group (point-form dynamics), the three-dimensional Euclidean group (instant-form dynamics), and the subgroup of the Poincaré group that leaves a hyperplane

$$
x^{+}:=x^{0}+\hat{\mathbf{z}} \cdot \mathbf{x}=0
$$

tangent to the light cone invariant (light-front dynamics). This last subgroup is a seven-parameter subgroup, while the Lorentz group and three-dimensional Euclidean groups are six-parameter subgroups. Relativistic quantum theories where there are no interactions in the generators of this seven-parameter subgroup are called light-front quantum theories. Light-front representations have the smallest number of generators that require interactions.

The seven-parameter subgroup that leaves the light-front hyperplane invariant includes (1) a three-parameter subgroup of translations tangent to the hyperplane (2) a three-parameter subgroup of light-front preserving Lorentz boosts and (3) a one-parameter subgroup of rotations about the longitudinal, $(\hat{\mathbf{z}})$, axis. In the $S L(2, \mathbb{C})$ representation the light-front Lorentz transformations are represented by the subgroup of lower triangular matrices:

$$
\begin{gathered}
\Lambda_{f b}(p / m)=\left(\begin{array}{cc}
\sqrt{p^{+} / m} & 0 \\
p_{\perp} / \sqrt{p^{+} m} & \sqrt{m / p^{+}}
\end{array}\right) \quad \text { light-front boosts } \\
\Lambda_{f r}(\phi)=\left(\begin{array}{cc}
e^{i \phi / 2} & 0 \\
0 & e^{-i \phi / 2}
\end{array}\right) \quad \text { rotations about } \hat{\mathbf{z}}
\end{gathered}
$$

where the corresponding $4 \times 4$ Lorentz transformations are $\Lambda^{\mu}{ }_{v}=\frac{1}{2} \operatorname{Tr}\left(\sigma_{\mu} \Lambda \sigma_{v} \Lambda^{\dagger}\right)$. The generators of the transverse boosts and the longitudinal rotations also satisfy the commutation relations of the two-dimensional Euclidean Lie algebra.

The light-front Hamiltonian, $P^{-}$, is one of the three dynamical generators. It satisfies the light-front dispersion relation

$$
P^{-}:=H-\hat{\mathbf{z}} \cdot \mathbf{P}=\frac{\mathbf{P}_{\perp}^{2}+M^{2}}{P^{+}} .
$$

One property that distinguishes a light-front dynamics from Dirac's other forms of dynamics is that a light-front hyperplane is not a suitable initial value surface because it has light-like tangent vectors.

In order to compare spins of particles in different inertial frames, it is useful to boost to a common frame where the particle's spins can be compared. The frame most often used is the 
particle's (or system's) rest frame, but the spin defined this way depends on the boost used to transform to the rest frame, since a boost right multiplied by a rotation is still a boost. The lightfront spin is defined using light-front preserving boosts:

$$
S_{f}^{i}=\frac{1}{2} \sum \varepsilon_{i j k} \Lambda_{f b}^{-1}(P / M)^{j}{ }_{\mu} \Lambda_{f b}^{-1}(P / M)^{k}{ }_{v} J^{\mu v}
$$

where $J^{\mu v}$ is the angular momentum tensor, and the parameters, $P / M$, in the boosts are operators. Because the light-front preserving boosts form a subgroup, they cannot generate Wigner rotations. This means that light-front boosts leave the light-front magnetic quantum numbers unchanged.

One interesting property of a light-front dynamics is that the rest frame is defined by the dynamical constraint $P^{-}=P^{+}=M$. This means that the conditions that determine the rest frame of free and interacting systems do even commute. This has dynamical implications for the spins, because even though the light-front boost are kinematic, the rest frame, where spins in different frames are compared, is dynamical.

A basis for a particle of mass $m$ and spin $s$ can be constructed out of simultaneous eigenstates of the mass, spin, and four other mutually commuting functions of the Poincaré generators. They can be taken as the generators of translations tangent to the light-front hyperplane and the longitudinal component of the light-front spin:

$$
M^{2}, S^{2}, \underbrace{P^{+}, P^{1}, P^{2}}_{\tilde{\mathbf{P}}}, S_{f}^{3} .
$$

The single-particle basis vectors are

$$
|(m, s) \tilde{p}, \mu\rangle .
$$

Lorentz covariance of the four-momentum and angular-momentum tensors determine both the transformation properties and spectra of these observables. The resulting transformation property of a mass $m$ spin $s$ irreducible representation of the Poincaré group is:

$$
U(\Lambda, a)|(m, s) \tilde{p}, \mu\rangle=e^{i p^{\prime} \cdot a} \sum_{v}\left|(m, s) \tilde{p}^{\prime}, v\right\rangle \sqrt{\frac{p^{+\prime}}{p^{+}}} D_{v \mu}^{s}\left[\Lambda_{f b}^{-1}\left(p^{\prime} / m\right) \Lambda \Lambda_{f b}(p / m)\right]
$$

where $\Lambda_{f b}(p / m)$ are light-front boosts from the rest frame to $\tilde{\mathbf{p}}$ and $p^{\prime}=\Lambda p$.

While the light-front spin is invariant with respect to light-front boosts, it undergoes Wigner rotations under ordinary rotations. More importantly, the light-front Wigner rotation of a rotation is not the rotation. What this means is that if a system of non-interacting particles is rotated, the spin of each particle will rotate with a different angle that depends on the particle's momentum. This means that light front-spins cannot be added with ordinary $S U(2)$ Clebsch-Gordan coefficients.

In order to add light-front spins for systems of free particles it is necessary to boost to the system rest frame, transform the light-front spins to canonical spins, add the canonical spins and orbital angular momenta, then boost the back to the original frame with a light-front boost. The coefficients of the resulting unitary transformation are the Clebsch-Gordan coefficients for the Poincare group in a light-front basis. The rotations that transform the light-front spins to canonical spins, are called Melosh rotations. They involve a light-front boost followed by an inverse rotationless (canonical) boost,

$$
R_{M}(p / m)=\Lambda_{c b}^{-1}(p / m) \Lambda_{f b}(p / m) .
$$


It is more convenient to treat systems of particles in a many-body basis where the internal degrees of freedom are invariant with respect to light-front boosts. These variables include the total light-front momenta, light-front momentum fractions, the transverse single-particle three momenta transformed to the kinematic rest frame with light-front boosts, and the light-front magnetic quantum numbers. These variables have the property that only the total light-front momentum transforms under light-front boosts; all of the other variables are invariant.

Dynamical light-front models can be constructed by adding kinematically invariant interactions to the non-interacting light-front Hamiltonian

$$
P^{-} \rightarrow\left(\mathbf{P}_{\perp}^{2}+M_{0}^{2}+V\right) / P^{+}
$$

of a many-particle system. Allowable interactions must preserve the spectral condition and result in a self-adjoint $P^{-}$. Rotational covariance is an additional non-trivial dynamical constraint. These conditions can be realized in few-body models. When $P^{-}$is self-adjoint the dynamics is welldefined and given by a one-parameter unitary group, so in this case the self-adjointness of the light-front Hamiltonian ensures that there is no ambiguity associated with the bad initial value surface.

The Light-front representation has advantages for computing current matrix elements. There are several reasons for this. First, since the boosts are kinematic, boosts of the initial and final states can be computed by applying the inverse boost to arguments of the light-front wave function, which is normally expressed in a non-interacting multi-particle basis. In addition, because the light-front boosts form a subgroup, the light-front spins in the initial and final states of the the current matrix elements are frame independent. If the momentum transfer is space-like, the orientation of the light front can be adjusted so all current matrix elements can be expressed in terms of matrix elements of $I^{+}(0)$, and these matrix elements are invariant under light front-boosts. Another unique feature of the light-front representation is that for one-body (impulse) current operators the momentum transferred to the system is the same as the momentum transferred to the constituents in all frames related by light-front boosts. This is not the case in Dirac's instant or point-form dynamics, however in the light-front case for spin greater than $1 / 2$ there are more current matrix elements than there are independent form factors. The additional current matrix elements are related by dynamical rotational covariance.

A true covariant current in a dynamical model cannot be a one-body operator and satisfy current covariance and current conservation. Impulse approximations can be made by assuming that the two-body current vanishes on a set of independent matrix elements. The remaining matrix elements can be computed by imposing rotational covariance. This is not as satisfactory as having a covariant current operator that can be used with different initial and final states.

One feature of Dirac's different representations of the dynamics is that they are all scattering equivalent (related by an $S$-matrix preserving unitary transformation). This means that given generators in one representation it is possible to find equivalent generators in any other representation. This equivalence suggests that it is useful to exploit the advantages of the light-front representation.

The interest in the light-front representation is motivated by properties of the light-front formulation of quantum field theory. For free fields, light-front fields are constructed by changing variables from three momenta to the three light-front components of the four momentum. One feature of the light-front field is that a canonical momentum field is not needed to separate the creation 
and annihilation operators:

$$
a(\tilde{\mathbf{p}})=\sqrt{\frac{p^{+}}{2}} \theta\left(p^{+}\right) \phi\left(x^{+}=0, p^{+}, \mathbf{p}_{\perp}\right) \quad a^{\dagger}(\tilde{\mathbf{p}})=\sqrt{\frac{p^{+}}{2}} \theta\left(p^{+}\right) \phi\left(x^{+}=0,-p^{+}, \mathbf{p}_{\perp}\right) .
$$

What this means is that any operator that commutes with the field restricted to a light front must be a constant multiple of the identity. This means that free fields restricted to the light front are irreducible.

A related property of the free fields restricted to the light front is that the fields are independent of the mass. For canonical fields, the canonical transformation that changes masses cannot be implemented by a unitary transformation [3], while fields restricted to a light front are trivially related by a unitary transformation [4].

Another property of light-front field theory follows because $P^{+}$is a kinematic operator satisfying the spectral condition $P^{+} \geq 0$. Interactions that preserve the kinematic symmetries must commute with $P^{+}$. This means that $V|0\rangle$ is an eigenstate of $P^{+}$with eigenvalue 0 . It follows that

$$
\langle 0|V V| 0\rangle=\langle 0|V| 0\rangle\langle 0|V| 0\rangle,
$$

because there can be no contribution from states with absolutely continuous spectrum of $P^{+}$since $P_{i}^{+}=0$ is a set of measure 0 . This requires $V|0\rangle=|0\rangle\langle 0|V| 0\rangle$. Invariance of the vacuum can be realized by a simple constant renormalization of $P^{-}$, with the vacuum remaining the Fock vacuum.

The role of the Fock-vacuum in light-front dynamics can be best understood by considering Noether's theorem on the light front. The Poincaré invariance of the action leads conserved Noether currents. Integrating the "+" components of these currents over the light front, assuming no contributions from the boundaries, means that all 10 Poincaré generators are independent of $x^{+}$. The expression for these generators involves fields on the light front and derivatives of these fields. While the fields and derivatives tangent to the light front are all in the irreducible light-front algebra, the derivatives of the fields normal to the light front are unconstrained.

This ambiguity is related to the problem with the inadequacy of the light front as an initial value surface. This does not mean that the irreducibility cannot be exploited. It means the additional information is needed to define the derivatives off of the light-front in terms of the irreducible algebra on the light front. For a scalar field theory all of the normal derivatives cancel. In this case all the generators can be expressed in terms of the operators in the irreducible light front algebra. The dynamical generators have the form

$$
\begin{gathered}
K^{3}=\int_{x^{+}=0} d \tilde{\mathbf{x}}:\left(4 \frac{\partial \phi(x)}{\partial x^{-}} \frac{\partial \phi(x)}{\partial x^{-}} x^{-}-\left(\nabla_{\perp} \phi(x) \cdot \nabla_{\perp} \phi(x)+m^{2} \phi(x) \phi(x)+V(\phi)\right) x^{+}\right): \\
P^{-}=\int_{x^{+}=0} d \tilde{\mathbf{x}}:\left(\nabla_{\perp} \phi(x) \cdot \nabla_{\perp} \phi(x)+m^{2} \phi(x) \phi(x)+V(\phi)\right): \\
\left.F^{i}=\int_{x^{+}=0} d \tilde{\mathbf{x}}:\left(\left(\nabla_{\perp} \phi(x) \cdot \nabla_{\perp} \phi(x)+m^{2} \phi(x) \phi(x)+V(\phi)\right) x^{i}+2 x^{-} \frac{\partial \phi(x)}{\partial x^{-}} \frac{\partial \phi(x)}{\partial x^{i}}\right)\right): .
\end{gathered}
$$

$K^{3}$ has a dynamical component, but it is multiplied by $x^{+}$which vanishes on the light front. Iterating the light-front Heisenberg field equations, assuming that the commutation relations of the fields on the light front agree with the free-field commutation relations,

$$
\left[\phi\left(\tilde{\mathbf{x}}, x^{+}=0\right), \phi\left(\tilde{\mathbf{y}}, y^{+}=0\right)\right]=i \frac{\pi}{4} \delta\left(\mathbf{x}_{\perp}-\mathbf{y}_{\perp}\right) \varepsilon\left(x^{-}-y^{-}\right)
$$


results in a series in $x^{+}$with coefficients that involve fields and commutators of fields restricted to the light front.

The expressions for the dynamical generators have the usual diseases in that products of fields at the same point are ill-defined. For the case of free fields the light-front Heisenberg equations are linear and can be solved, resulting in the correct expression for the field operator of mass $m$ in the light-front representation. Vacuum expectation values of products of these fields, no longer restricted to the light front, in the trivial vacuum give the Wightman functions of the free field theory. These are moments of the dynamical (mass-dependent) vacuum. While on one hand this result is trivial, free fields with different masses live on different Hilbert spaces with different vacuua. This shows that the correct result can still be obtained by using the trivial vacuum and the light-front field algebra with different interactions.

In an interacting field theory additional information is necessary to define the theory. This involves finding a non-perturbative way to define products of fields at the same point. Normally non-trivial vacuua are due to the all creation operator terms in the Hamiltonian. In the case of a $\phi^{4}(x)$ theory the creation operator part of the light-front $P^{-}$has a term with general form

$$
\begin{gathered}
\int \frac{\theta\left(p^{+}\right) \delta\left(p^{+}\right) d p^{+}}{\left(p^{+}\right)^{2} \prod \xi_{i}} \prod d \mathbf{p}_{i \perp} d \xi_{i} \delta\left(\sum \mathbf{p}_{i \perp}\right) \delta\left(\sum \xi_{i}-1\right) \times \\
a^{\dagger}\left(\xi_{1} p^{+}, \mathbf{p}_{\perp 1}\right) a^{\dagger}\left(\xi_{2} p^{+}, \mathbf{p}_{\perp 2}\right) a^{\dagger}\left(\xi_{3} p^{+}, \mathbf{p}_{\perp 3}\right) a^{\dagger}\left(\xi_{4} p^{+}, \mathbf{p}_{\perp 4}\right) .
\end{gathered}
$$

While formally the $\delta\left(p^{+}\right)$suggests that the vacuum should remain trivial, this contribution is illdefined (and very singular) at $p^{+}=0$. The fate of the trivial vacuum depends on what replaces this operator. The singularities at $p^{+}=0$ are not independent of the ultraviolet singularities of the theory, since they are transformed into each other as the orientation of the light front is rotated. Both need to be addressed in order to construct a self-adjoint $P^{-}$.

When the theory has infrared singularities, additional contributions concentrated at $P^{+}=0$ may be required to maintain equivalence with the canonical theory. These affect the interpretation of the light-front vacuum. These zero-modes are required in perturbation theory. While these zero modes disappear in a theory with cutoffs; it is not clear if there is a way to recover them as the cutoff is removed. or if they have to be put in by hand.

\section{References}

[1] Wigner, Eugene P., On Unitary Representations of the Inhomogeneous Lorentz Group, Annals Math. 40(1939)149.

[2] Dirac, Paul A. M., Forms of Relativistic Dynamics, Rev. Mod. Phys. 21(1949)392.

[3] Haag, R., On quantum field theories, Kong. Dan. Vid. Sel. Mat. Fys. Med., 29N12(1955)1.

[4] Leutwyler, H., Klauder, J. R. and Streit, L, Quantum field theory on lightlike slabs, Nuovo Cimento., A66(1970)536. 